\section{REACTIVAR LA DEMOCRACIA, UN DESAFÍO ÉTICO Y EDUCATIVO: REFLEXIONES URGENTES A PARTIR DE LA OBRA DE JOHN DEWEY}

\author{
Antonio Luzón Trujillo \\ Universidad de Granada \\ ORCID iD: https://orcid.org/0000-0002-4095-2319 \\ aluzon@ugr.es \\ Juan Carlos González Faraco \\ Universidad de Huelva \\ ORCID iD: https://orcid.org/0000-0003-2004-7706 \\ faraco@uhu.es
}

Cómo citar este artículo/Citation: Luzón Trujillo, A.; González Faraco, J. C. (2019). Reactivar la democracia, un desafío ético y educativo: reflexiones urgentes a partir de la obra de John Dewey. Arbor, 195 (792): a512. https://doi.org/10.3989/ arbor.2019.792n2013

Recibido: 25 junio 2018. Aceptado: 11 julio 2018

RESUMEN: Este artículo plantea una relectura de John Dewey con la intención de analizar y dar algunas respuestas a la actual crisis de la democracia representativa o liberal. Esta crisis guarda relación con procesos globalizadores contradictorios que, de un lado, alimentan una utopía futurista confiada en el progreso tecnológico, y de otro, el regreso "retrotópico", nostálgico y emocional, a lo tribal. Esta relectura se centra en obras fundamentales de Dewey, pero especialmente Democracia y Educación y otros textos de carácter pedagógico. El artículo desarrolla una serie de argumentos que concluyen en torno a la idea de "democracia creativa", que Dewey planteó en su madurez, como referencia para una deseable reactivación de la democracia entendida como ideal ético.

PALABRAS CLAVE: John Dewey; democracia; educación; democracia creativa; educación democrática; ciudadanía; globalización.

\section{REACTIVATING DEMOCRACY, AN ETHICAL AND EDUCATIONAL CHALLENGE: URGENT REFLECTIONS BASED ON THE WORK OF JOHN DEWEY}

Copyright: (C 2019 CSIC. Este es un artículo de acceso abierto distribuido bajo los términos de la licencia de uso y distribución Creative Commons Reconocimiento 4.0 Internacional (CC BY 4.0).
ABSTRACT: This article proposes a rereading of John Dewey with the intention of analyzing and proposing some solutions to the current crisis of representative or liberal democracy. This crisis is related to contradictory globalizing processes that, on the one hand, nourish a futuristic utopia entrusted to technological progress, and on the other, regresses to the nostalgic, emotional and tribal. This rereading focuses on some foundational works of Dewey, but especially on Democracy and Education and other educational texts. The article develops a series of arguments that revolve around the idea of «creative democracy", which Dewey developed in his maturity, as a valuable reference for a desirable reactivation of democracy, understood as an ethical ideal.

KEYWORDS: John Dewey; democracy; education; creative democracy; democratic education; citizenship; globalization. 


\section{LEER A JOHN DEWEY HOY}

La democracia liberal parece haber entrado en una preocupante decadencia en este siglo XXI. A esta sombría conclusión han llegado numerosos observadores de diversos campos de conocimiento y gran relieve intelectual, como Zigmunt Bauman, George Steiner, Martha Nussbaum, Byung-Chul Han y Francis Fukuyama, entre otros. No se trata, desde luego, de una opinión unánime, pero sí muy extendida, expresada con gradaciones y matices múltiples. Sin embargo, es innegable que hay señales suficientemente significativas de que la globalización económica no le está siendo particularmente favorable a la democracia, ni tampoco, especialmente, el veloz y apabullante avance de la sociedad digital. En realidad, la nueva utopía futurista, animada y regida por la razón tecnológica, con su saga de big data, redes sociales, vigilancia y control, consumo hipertrofiado, vida líquida, individualismo y hegemonía de la apariencia, ha debilitado los cimientos éticos y dislocado las referencias fundamentales de la democracia hasta ahora conocida (Bauman y Lyon, 2013; Han, 2012; Han, 2013; Han, 2014; Nussbaum, 2010). Por si eso fuera poco, como una reacción supuestamente contraria y acaso previsible, esta utopía se está viendo acompañada aquí y allá por movimientos "retrotópicos" (Bauman, 2017) que se alimentan de la nostalgia de paraísos perdidos, de incertidumbre y temor, y que por ello se obcecan en "la expulsión de lo distinto", como diría Han (2017). Estos movimientos, que en Europa no dejan de proliferar e inquietarnos (Han, 2017, pp. 25-26; Steiner, 2012), alientan el sentido tribal y están haciendo florecer otra vez los nacionalismos, con su retórica identitaria y emocional, en la que no faltan -aunque se disimulen- el resentimiento (Fukuyama, 2019), el etnicismo, la xenofobia y el dogma político, y que ha encontrado en la hipercomunicación un aliado eficacísimo para su autoproducción y reproducción a gran escala (Bauman, 2017).

En este estado de cosas, de cierta desafección por la democracia y preocupante decaimiento de sus valores radicales, en favor de formas de psicopolítica que crean ilusión de libertad (Han, 2015a), ¿tiene algún sentido recurrir a un intelectual como John Dewey y, entre otras, a una obra como Democracia y educación, publicada hace más de cien años (1916)? ¿Qué podemos aprender de Dewey para comprender mejor nuestro tiempo y tratar de reanimar la democracia y revitalizar el sentido profundo de la ciudadanía? ¿Qué papel le tocaría desempeñar a la educación en este proyecto de regeneración social y política? (Gordon y English, 2016). A estas preguntas va a intentar responder, o al menos ensayar algunas respuestas o sugerencias, este artículo.

La relación, íntima y solidariamente provechosa, entre la democracia y la educación es capital en la obra de John Dewey. Ambas se potencian entre sí y adquieren juntas un significado que trasciende la dialéctica individuo-sociedad. Ya en Mi credo pedagógico (1897), uno de sus primeros textos, Dewey consideraba que la institución escolar constituye una forma de vida en comunidad, un microcosmos cultural y político, y que el proceso educativo, que es parte sustantiva de la vida y no una simple transición hacia el futuro, se expresa precisamente en una doble dimensión individual y social. Una buena educación democrática no debe tener como exclusiva referencia una determinada organización institucional de la gestión política, sino más bien un modelo de vida inspirado en un ideal, que se realiza a través de la experiencia humana en sociedad mediante el intercambio y el enriquecimiento mutuo constante. “Una democracia -escribía en 1916- es más que una forma de gobierno, es primariamente un modo de vivir asociado, de experiencia comunicada juntamente" (Dewey, 1916/1995, p. 82).

Para John Dewey no hay democracia sin ciudadanos bien educados pero la democracia también es (o debiera ser) educadora en sí misma, una idea en la que se percibe la influencia de Hegel y, en concreto, su concepción del Estado y su noción del patriotismo como convicción política en la recíproca necesidad entre lo general, el Estado, y lo particular, el ciudadano singular, quien adopta esa convicción en uso de su conciencia libre (Filosofía del derecho, 1821, sección III, §267, §268 y §269). Por desgracia, la democracia se vuelve con cierta asiduidad una fórmula retórica, vacía de contenido. Dewey, a quien muchos consideran el filósofo de la democracia estadounidense (Hook, 2000), le confiere, en cambio, un hondo significado, con sólido fundamento hermenéutico, que representa toda una filosofía social, según la cual el individuo contribuye activamente, mediante su experiencia, a la construcción del ideal democrático. Estamos, pues, ante una concepción del sujeto que se asienta en la relación de reciprocidad entre individuo y sociedad. De esta relación, es decir, de la experiencia democrática como forma de vida, nacen la esperanza y la fe en la capacidad del ser humano para crear una comunidad moral y política.

En toda la obra de John Dewey destaca la idea de una democracia participativa, entendida como pro- 
ceso de intervención activa compartida por todos los agentes que conforman la comunidad a cualquier escala. Esta idea pervive y ha prosperado en la obra de prestigiosos autores para los que Dewey sigue siendo un valioso referente, como Apple y Beane (1997), Giroux (2006), Guttman (2001), Popkewitz (2003), Popkewitz (2008), entre otros estudiosos de la educación, o como Putnam y Putnam (1993), Rorty (2000), Sartori (2007) o Habermas, entre los filósofos.

Dewey y su obra representan, de ahí su apremiante actualidad, una clara apuesta por la democracia, no únicamente como sistema o mecanismo político, sino como dilema moral que realza el valor de lo público y de la justicia social. Para Thomas Popkewitz (2003), por ejemplo, sus aportaciones teóricas son tan relevantes antes como ahora, pues ayudan a repensar la epistemología educativa y, por ende, el papel de la educación en lo que se refiere a los cambios históricos que se han producido en los modernos procesos de administración social del individuo, es decir, en las trayectorias y estrategias colectivamente seguidas para fabricar a lo largo del tiempo identidades y sistemas de exclusión e inclusión social.

John Dewey no solo es un clásico eminente de la pedagogía progresiva o de la filosofía pragmatista norteamericana. Sus escritos resuenan todavía como una preciosa herencia para el pensamiento de nuestra época y, en particular, para la filosofía de la educación (BellataIla, 2016). También es cierto que su obra es inevitablemente hija de su tiempo, una etapa histórica de intensa industrialización, urbanización y racionalización, y responde en buena medida a la fe en el progreso social y en los valores que representa la modernidad. A lo largo de este texto, trataremos, sin embargo, de descubrir qué nos puede decir a estas alturas, en tiempos de globalización y posmodernidad, la obra de John Dewey.

Cuando con razón se insiste, desde hace tiempo, en que la educación vive una etapa crítica $^{1}$, a causa de los imperativos de la cultura del rendimiento, la invasión de criterios económicos y tecnológicos y la consecuente postergación de las humanidades (Lledó, 2014, pp. 26-28; Nussbaum, 2010), su relectura se vuelve cada vez más sugestiva y necesaria. Dewey nos advierte de que lo esencial no reside en los métodos o en el modelo pedagógico (más o menos directivo, o bien adaptado a las motivaciones de los alumnos), sino en el modelo de sociedad y de pensamiento social: en cómo hacer posible una comunidad política libre y justa. Beltrán Llavador (2004) en una esclarecedora síntesis alude a tres importantes razones para releer a John Dewey:
En primer lugar [...], frente al viejo trascendentalismo esencialista que nos instaló en categorías dualistas, opone el optimismo de una apuesta decidida por los seres humanos y el protagonismo de la educación, lo que supone una apelación a la responsabilidad individual y colectiva. En segundo lugar, la importancia concedida al método experimental que ciertos abusos, pero sobre todo una ignorancia nada inocente, han conseguido arrumbar como exclusivo de individuos muy especializados en áreas científico-técnicas. En tercer lugar, y en estos momentos tan poco desdeñable como los dos anteriores, Dewey rescata el valor social y político de la educación con lo que se redimensiona la tarea escolar ${ }^{2}$ (p. 2).

Como González Monteagudo (2001) señala, el legado teórico de John Dewey supone, a pesar de las críticas de la Escuela de Frankfurt ${ }^{3}$ a su pragmatismo identificándolo con el positivismo, una herramienta útil para pensar y actuar en los nuevos escenarios socioculturales y educativos por su originalidad, profundidad y polivalencia. Nubiola y Sierra celebran el redescubrimiento de Dewey y le auguran un creciente impacto en España y Lationamérica. Vaamonde Gamo y Nubiola (2016) resaltan, por ejemplo, su influencia en el feminismo contemporáneo, sobre todo norteamericano. $Y$ naturalmente son muchos los estudiosos de su obra que ponderan sus aportaciones a la renovación pedagógica y lo sitúan entre los más grandes e influyentes teóricos de la educación y educadores del siglo XX (por ejemplo, Igelmo, Fuentes y Jover, 2017; Jover y García Fernández, 2015; Jover y del Pozo, 2013; Rogacheva, 2016). Hay, en fin, una coincidencia general en señalar la extraordinaria circulación internacional de sus ideas (Bellatalla, 2016; Jover, RuizValdepeñas y Thoilliez, 2010) y en valorar la vitalidad de su obra, especialmente de Democracia y educación ${ }^{4}$.

\section{LA EDUCACIÓN, PUENTE ENTRE LA CULTURA Y EL INDIVIDUO}

En “¿Qué es educación?”, primer texto de Mi credo pedagógico (1897), Dewey recuerda el vínculo indisociable, orgánico, como él mismo lo denomina, entre individuo, educación y sociedad. A su juicio, la educación vendría a ser el proceso de participación del individuo en la "conciencia social", un proceso que arranca desde el nacimiento, "saturando su conciencia y formando sus hábitos" (cit. en Dewey, 2009, p. 41).

En esta primera etapa, Dewey distingue entre los procesos educativos inconscientes e incidentales y la educación como proceso inacabado, guiado por objetivos que establece la sociedad. De ese modo, concibe la educación como una acción dinámica vinculada a 
un determinado orden social que la comunidad legitima. Domingo Barnés ${ }^{5}$, en el prólogo de La escuela y la sociedad (1899/1929), subrayaba el significado social de la educación en la obra de John Dewey: "Lo que llaman 'la nueva educación' no es sino un intenso florecimiento de tendencias y trabajos para purificar toda la vida social".

Aunque el pensamiento pedagógico de Dewey se encontraba aún en sus albores, ya Barnés percibía en él este prioritario carácter social de la educación, que nunca debería llegar a reducirla a un simple bien instrumental con un objetivo económico. En esta obra, La escuela y la sociedad (publicada originalmente en 1899), Dewey deja algunas muestras de su pensamiento antiutilitarista y hace una sutil crítica a la política educativa dominante, mostrándose a favor de una educación basada en la experiencia (learning by doing), lo que además potencia su proyección social:

[...] mientras nuestros directores de la política pedagógica hablen de la cultura, del desenvolvimiento de la personalidad, etc., como los fines y aspiraciones de la educación, la gran mayoría de los que pasan bajo la tutela de la escuela la consideran como un estrecho instrumento práctico para satisfacer sus exigencias de una vida restringida. Si introducimos en el proceso educativo las actividades que interesan a aquellos cuyo interés predominante es hacer y obrar, veremos que el arraigo de la escuela entre sus miembros es más vital y en el fondo más cultural (Dewey, 1899/1929, pp. 46-47).

Sin embargo, es en Democracia y educación, aunque no solo, donde Dewey (1916/1995) enfatiza la función social de la educación y su sentido vital, definiéndola como "necesidad de vida", encrucijada donde se dan la mano filosofía y educación. Esta es acción, vida misma; comporta un modelo antropológico y, en consecuencia, es objeto de la filosofía:

Como la educación es el proceso mediante el cual puede realizarse la transformación necesitada y no seguir siendo una mera hipótesis deseable, alcanzamos una justificación de la afirmación de que la filosofía es la teoría respecto a lo que es la educación, entendida como una práctica dirigida (p. 350).

Una idea que recorre toda la obra de Dewey es la de la "reconstrucción continua de la experiencia", basada en un método inquisitivo libre de prejuicios ideológicos o religiosos y, por ende, laico (Bellatalla, 2016, p. 25). Con esta idea se distancia de una concepción educativa formalista, basada en el adiestramiento de las facultades del espíritu que el sujeto trae consigo al nacer. El valor de la experiencia individual en su interacción con el medio acentúa la importancia de la experiencia social como proceso dinámico y permanente que se abre a y crea sucesivas experiencias encadenadas entre sí, lo que facilita la interpretación del presente y contribuye a mejorarlo (Cadrecha Caparrós, 1990; Putnam y Putnam, 1993). A este respecto, Atkinson (2017) señala que, según Dewey, tanto en Democracia y educación como en Experiencia y educación, el objetivo de la educación

[...] no debería ser principalmente perpetuar el statu quo, simplemente transmitiendo conocimiento y procesos históricos, sino más bien una combinación de transmisión cultural y una crítica a nuestra historia que involucre y lleve a los estudiantes hacia una sociedad más ilustrada, democrática y justa. Sólo entonces puede ser útil para que la sociedad avance en una dirección positiva (p. 5).

Así pues, para John Dewey la educación reuniría un doble significado. De un lado, uno amplio y comprensivo, en cuanto que abarca un conjunto de procesos mediante los cuales un grupo transmite a sus miembros más jóvenes aquellos ideales que aseguren su supervivencia. En ese sentido, la educación sería, como señalaba Atkinson (2017), un proceso de transmisión y adquisición cultural. Del otro, uno más restringido, en el que confluyen e interactúan dos vertientes, la psicológica y la social. En suma, el individuo y la cultura.

Fiel a la tradición hegeliana, toda la teoría educativa desarrollada por John Dewey pretende la superación de dualidades, tantas veces reduccionistas, como la disyuntiva entre "lo individual" y "lo social" (Good y Garrison, 2010; Sarofian-Butin, 2017). A partir de su concepción "interaccionista" de la experiencia, en la que causa y efecto son caras de la misma moneda, la educación garantiza la pervivencia de la sociedad mediante una relación de intercambio y enriquecimiento mutuo entre individuo y grupo. Cada persona se educa, pues, gracias a experiencias individuales y colectivas en su medio cultural. Sin embargo, tanto el yo individual, en cuanto unidad dinámica constituida por un sujeto consciente de sí y de su relación con el medio, como lo social se conjugan en plural, presentan identidades múltiples. La individualidad cobra sentido en el marco de la vida social, con su vasta pluralidad de formas de asociación humana, mediante un intercambio continuo de experiencias con los otros y con las cosas de su entorno. En palabras de Dewey (1927/2004), "la afirmación de que los individuos viven en un mundo, significa en concreto que viven una serie de situaciones, lo que significa que hay una interacción entre individuos, ob- 
jetos y otras personas" (p. 86). Interacción y continuidad son, en su pensamiento, principios inseparables.

La experiencia educativa no se limita exclusivamente al desarrollo cognitivo. Permite la adaptación a nuevas experiencias de diverso género y en diversas direcciones. Es ante todo una experimentación activa con el mundo, por lo que el aprendizaje y la integración social no se oponen ni obstaculizan el aprendizaje y el desarrollo individual. Todo lo contrario: la sociedad forma individualidades que a su vez mejoran la vida colectiva. Es la comunidad, cada comunidad, la que, de hecho, hace posible la emergencia de proyectos e identidades individuales. En ese contexto, la educación sería un modo de reconstruir la experiencia favoreciendo el engarce entre la experiencia individual y colectiva, social y política (Chanial, 2006). O, dicho de otro modo, haciendo posible la narratividad de las experiencias, cuestión esta crítica si tenemos en cuenta el narcisismo de muchas conductas, además de la aceleración y atomización del tiempo, en la sociedad líquida actual (Han, 2013; Han, 2014; Han, 2015b; Tolentino Mendonça, 2017).

\section{LA DEMOCRACIA, SISTEMA POLÍTICO, COMUNIDAD MORAL Y AGENTE EDUCATIVO}

Que la educación forma parte del proyecto político no es una novedad. Ya en la democracia ateniense se tenía a la polis por una verdadera instancia educadora y a la paideia como uno de sus baluartes. Para Dewey la educación adquiere significado en y para una sociedad democrática, una forma de asociación específicamente humana regida por un "ideal ético" y por ello alejada de un sentido organicista que solo la ve como amalgama de individuos que se conducen aisladamente. Más que una forma particular de gobierno, la democracia es un modo de vida, una práctica que confronta el conocimiento y las actitudes de hombres y mujeres en su convivencia diaria.

Como testigo de tantos y tan importantes procesos históricos y como intelectual socialmente comprometido, Dewey tuvo innumerables ocasiones para observar la "tragedia" en la que se debate el hombre contemporáneo en la consecución de sus fines o en la búsqueda de su propia seguridad. Desde su punto de vista, el ideal democrático anhela parigualmente el perfeccionamiento del individuo y de la sociedad, pues procura la mejora de las capacidades de cada uno de sus miembros. Para ello adopta una perspectiva integradora que se encarna, se realiza en la experiencia (democrática) cotidiana. Sin ella, no hay democracia, solo un sistema frío sin cometido moral y un proyecto colectivo ilusorio... En su ideal democrático John Dewey destaca la lucha contra las desigualdades, sociales o escolares, asociadas a la división de clases, raza, género o a otros sistemas de jerarquía social (Vaamonde Gamo y Nubiola, 2016). También es contumaz en su defensa de la libertad intelectual, que posibilita la realización personal junto al otro, y que proviene de su universo ilustrado y liberal.

El pragmatismo, en efecto, constituye un proyecto de diseño de un individuo que abraza libremente las normas y los principios de la administración de la educación, de la ciencia y del progreso (Popkewitz, 2008). En su sistema de razón, a la educación le correspondería estimular y promover la libertad individual mediante la acción reflexiva, en consonancia con las voluntades colectivas (Guichot, 2003; Rubio Carracedo, 2009). Ciertamente, "democracia" y "liberalismo" son conceptos emparentados, si bien con ostensibles diferencias entre sí. Acaso la más palmaria sea el dispar acento que suelen poner en la igualdad y la libertad individual, respectivamente. Al liberalismo, en su sentido histórico, se le ha solido identificar con un espíritu abierto y tolerante, valores que se ven reflejados en la democracia representativa, por ser el sistema de gobierno que mejor protege la libertad individual, política y económica. A Dewey, sin embargo, esta concepción de la democracia siempre le pareció demasiado utilitarista. A su juicio, la asociación natural de los seres humanos tiende a ser caótica, por lo que suele derivar en una masa fragmentada y desorganizada que no atiende a ningún fin social, sino tan solo al interés individual (Geneyro, 1991). Entendida así, en sentido liberal estricto, la democracia quedaría circunscrita, según Dewey, a una modalidad de sistema político sujeto a reglas y normas cuyo objeto, aportar soluciones individuales, presupone, al modo hobbesiano, considerar al individuo como principal valor y como un fin en sí mismo (Rader, 1975).

El espíritu democrático de John Dewey responde, en cambio, a un ideal ético de vida en común. Y en él se dejan notar la influencia de Rousseau y del idealismo hegeliano, para los que la verdad debe buscarse en el conjunto, en un todo organizado y armónico en el que las partes encuentren sentido (Klenner, 2002). Ahora bien, Dewey (1927/2004), que no era, en absoluto, un colectivista, insiste continuamente en el juego dialéctico individuo-sociedad y valora el componente individual de toda acción colectiva. "Las personas individuales -escribía- son el centro de la acción, de la mental, de la externa y manifiesta. Las personas están sometidas a todo tipo de influencias sociales que de- 
terminan qué pueden pensar, planear y decidir" ( $p$. 97). Sin embargo, esta frase final, relativamente contradictoria con la anterior, recuerda en cierto modo las tesis del configuracionismo, pujante en la antropología cultural de esa época, y el determinismo social de ciertas escuelas herederas del positivismo cientificista del siglo XIX.

A Dewey se le ha vinculado habitualmente con el movimiento progresista norteamericano, aunque haya quienes discutan esta adscripción y lo tachen injustamente de conservador (Waddington, 2008). John Dewey fue un liberal de izquierdas (Waddington, 2008, p. 51), un socialdemócrata diríamos hoy, enemigo de todo dualismo simplificador (SarofianButin, 2017) y sumamente crítico con el estalinismo y con el New Deal conservador de su país (Rorty, 2000, p. 14). Pero fue además un reformador social (Bernstein, 2010) que quiso, durante toda su vida, con coherencia y perseverancia encomiables, ofrecer una respuesta democrática a los problemas de la sociedad americana, a través de la reforma de las costumbres y de los valores morales de los ciudadanos y de las instituciones (Castillo, 2003). De él son estas palabras cuya clarividencia y vigor se mantienen intactos hasta el día de hoy:

La grave amenaza a nuestra democracia no está en la existencia de estados totalitarios extranjeros, sino en la existencia dentro de nuestras propias actitudes personales y dentro de nuestras propias instituciones de condiciones semejantes a las que en otros países extranjeros han dado la victoria a la autoridad externa, a la disciplina, a la uniformidad y a la sujeción al líder. En consecuencia, el campo de batalla está dentro de nosotros mismos y de nuestras instituciones (Dewey, 1939/1965, pp. 48-49).

De ahí que, a su parecer, una buena sociedad solo pueda ser fruto de una buena educación. En el prefacio de Democracia y educación, Dewey ya apuntaba claramente esta idea, sobre la que girará toda la obra: "Las siguientes páginas representan un esfuerzo para descubrir y expresar las ideas contenidas en una sociedad democrática y aplicar estas ideas a los problemas de la obra educativa" (p. 8).

En cierto modo, la democracia se aprende en un proceso continuo de experiencia de vida en común. Educar es, en este sentido, mucho más que enseñar, rebasa la mera escolaridad y trasciende la razón pedagógica. Para Dewey (1935/1996) la democracia está regida por un "principio educativo" que se alimenta de la participación y de la experiencia compartida, por lo que "la tarea educativa no puede quedar reducida a la esfera puramente mental; no puede prescindir de una acción que produzca cambios reales en las instituciones" ( $p$. 43). Difícilmente puede entenderse el discurso intelectual de Dewey sin reconocer en él esa vieja creencia ilustrada, cada vez más retórica y difusa entre nosotros, en el poder transformador de la educación para lograr una organización más racional de la sociedad ${ }^{6}$.

\section{LA ESCUELA, LABORATORIO DE CIUDADANÍA}

En una de sus mejores obras, El sentido humanista del socialismo, Fernando de los Ríos (1926/1976) insistía en que el bienestar individual depende del de la comunidad, y en el papel que en ello juega la educación, cuyo valor encarecía con palabras como estas: "Sensibilizar es crear interés, ensanchar el ámbito de la curiosidad, sumirnos en el universo y dotarnos de una fina antena con que recoger las ondas del espíritu" (p. 251).

Lorenzo Luzuriaga (1968), otro institucionista, traductor y gran admirador de Dewey, iba incluso algo más allá: "La democracia será una farsa si los individuos no están preparados para pensar por ellos mismos, a juzgar independientemente, a ser críticos, a ser capaces de discernir las propagandas sutiles y los motivos que las inspiran" (p. 19).

En la misma sintonía, el preámbulo del decreto que estableció el nuevo plan de formación de maestros en $1931^{7}$ proclamaba lo siguiente: "El primer deber de toda democracia es éste: resolver plenamente el problema de la instrucción pública". Y continuaba después:

\footnotetext{
Urgía crear escuelas, pero urgía más crear maestros; urgía dotar a la escuela de medios para que cumpliera la función social que le está encomendada; pero urgía más capacitar al maestro para convertirlo en sacerdote de esta función; urgía elevar la jerarquía de la escuela, pero urgía igualmente dar al maestro de la nueva sociedad democrática la jerarquía que merece [...].
}

Dewey hubiera suscrito, sin dudarlo, una por una todas estas palabras, que vienen a avisarnos de que no puede haber democracia sin maestros bien formados y bien pagados, sin escuelas públicas de calidad, ni sin ciudadanos bien educados, cuyo talento y sensibilidad se proyecten benéficamente en la comunidad. Como asegura Geneyro (1991), lo que distingue a una comunidad de un aglomerado social, es precisamente compartir creencias y valores, que una verdadera educación para una ciudadanía democrática contribuirá a transferir y a afianzar de generación en generación. En tal empresa la escuela ocupa un locus social preferente. Ahora bien, 
entre la experiencia escolar y la cultura de su entorno pueden abrirse fisuras. Por ello, debería procurarse una fluida y equilibrada integración entre ambas para que el ideal democrático prospere como experiencia vivida y se convierta en un bien social. La escuela, como apuntaba Dewey en Democracia y educación, debería "buscar el equilibrio entre los diversos elementos del entorno social, y velar por que cada individuo tenga una oportunidad de escapar de las limitaciones del grupo social en el que ha nacido, y así entrar en contacto vivo con un entorno más amplio" (Atkinson, 2017, p. 5).

Fiel a su máxima del aprender haciendo y en total sintonía con su filosofía pragmática reformadora, Dewey entiende que las relaciones en el seno de la escuela, como comunidad social que es, deben estar regidas por principios democráticos, para cuya puesta en práctica la lógica escolar se vale, según Popkewitz (2003), de ciertas tecnologías del yo, bien conocidas y probadas, en lo que él denomina "gobernación del individuo" o "gobierno del alma". Según Popkewitz, el ciudadano moderno, en el pensamiento de Dewey, personifica una idea de individuo comprometido con la resolución de problemas comunes, próxima a la imagen actual del ciudadano que participa "voluntaristamente" en los asuntos sociales. En un trabajo posterior, ya citado, Inventing the Modern Self and John Dewey, Popkewitz (2008) describe la evolución del pragmatismo de Dewey, resaltando su gran influencia renovadora en diferentes culturas educativas, hacia lo que podría incluso definirse como un verdadero proyecto global, para el que la escuela pública, aunque no el único, es un espacio capital.

Para Dewey la escuela pública debe ser ante todo un laboratorio de ciudadanía (Bellatalla, 2016), una sociedad democrática a pequeña escala, porque enseñar la democracia sin practicarla sería, como él mismo sugería, algo así como aprender a nadar fuera del agua. Puede que valga la pena remontar el río y recuperar estas y otras ideas educativas de John Dewey. Tal vez nos ayuden a descifrar, interpretar correctamente y enfrentar la volatilidad de una cultura como la actual en la que, como asegura Bauman (2007), el conocimiento se ha vuelto "ese desconocido" y la educación es más "un producto" (en cierto modo, una cosa, una mercancía) que un proceso vital. Acaso convenga, como reivindica Amy Guttman (2001), pensar en la necesidad de una nueva teoría democrática de la educación en los aledaños de una educación política, compatible con el desarrollo de los valores democráticos, que afronte la banalización, el adormecimiento y la desafección que amenazan la salud de la democracia en nuestro tiempo.

\section{CONSIDERACIONES FINALES}

Algunos observadores actuales de la vida política, acaso más optimistas que otros a los que hemos hecho repetida alusión, como por ejemplo Victoria Camps (1990), están convencidos de que la voluntad y el compromiso ético de los ciudadanos pueden corregir el incierto rumbo de la "democracia imperfecta" que hoy vivimos con "la rectificación constante del error" (p. 32). Por su parte, Boaventura de Sousa Santos, en un lúcido trabajo titulado Globalización y democracia (2006), cree que la única alternativa para recobrar la dignidad en una sociedad indigna, que ha acabado con las expectativas y la esperanza de muchas personas, es la defensa a ultranza de los derechos humanos, lo que conllevaría la reinvención de la democracia.

En 1938, en una conferencia titulada Creative Democracy. The Task before $U s^{8}$, pronunciada durante un homenaje por su 80 cumpleaños, John Dewey ya advertía sobre la necesidad de crear un espacio público que hiciera posible el flujo y debate de ideas y proyectos, y la permanente adaptación a las nuevas circunstancias. Dewey confiaba en los frutos de una práctica real y efectiva de la ciudadanía o de lo que él llamó democracia creativa, aludiendo a un proceso de reflexión y deliberación. En aquellos años convulsos, desde la atalaya de una larga vida, Dewey veía los desmanes que asolaban la política internacional y, aun consciente de los graves peligros que acechaban a la democracia, creía con optimismo en su capacidad de recuperar el aliento, de regenerarse, siempre y cuando no se olvidara que "el territorio a conquistar no es físico sino moral“. Dewey no fía el porvenir de la democracia solo a la acción política y mucho menos a la retórica que suele acompañarla, sino al cambio de valores y actitudes, y por ello a la educación. Su optimismo está trufado de realismo. Nunca perdió la fe en la democracia durante la dura travesía de la política norteamericana en el periodo de entreguerras, pero era consciente de las grandes exigencias de orden moral que la democracia plantea a diario. "La democracia -escribió- se expresa en las actitudes que adoptan los hombres y se mide por sus consecuencias" (Dewey, 1938/2006, p. 254).

Además de un ethos revitalizado, John Dewey propone también una nueva estética que sustituya la coerción por la persuasión, la intolerancia por el diálogo, la incertidumbre por la convicción, el pesimismo por la esperanza. Todo ello prefigura un ideal de vida y una cosmovisión en los que la experiencia es decisiva ante las nuevas expectativas sociales. En plena concordancia con su filosofía entiende que la democracia se sostiene en la 
capacidad de convencimiento del ser humano, en sus actitudes y en su modo de vida, un todo que debe aunar la coherencia ética entre el pensamiento y la acción.

Dewey rehúye una fundamentación trascendente o exterior. Como si hubiera leído la máxima machadiana de "se hace camino al andar", piensa en la democracia como en un proceso de experiencia humana que analiza ideas y expresa las diferencias mediante el diálogo, poniendo en juego la inteligencia ciudadana convenientemente formada. Sin embargo, el ejercicio de las virtudes democráticas, como argumentaba Rawls (1996), no siempre conduce a un estado más perfecto ni puede arraigar ni fructificar en determinadas opciones políticas, como en los nacionalismos más extremos, de los que hubo muestras obscenas en el siglo XX y se aprecian signos alarmantes en el XXI (Álvarez Junco, 2016; Bauman, 2017; Fukuyama, 2019; Han, 2017; Steiner, 2012).

Ciertamente la democracia nunca es un hecho consumado, una estación término, una lección aprendida. Como un cuerpo vivo, ha de estar en permanente vigilia, hacer un alto y recuperar el aliento, avivarse a diario. Para su noción de "democracia creativa" Dewey apela al principio de responsabilidad individual, pero no tanto como un derecho o un deber ciudadano cuanto como un medio para mejorar la experiencia propia de vida y el desarrollo moral (Dewey, 1938/2006, pp. 252-253). No olvidemos que, si la experiencia es conocimiento, la experiencia democrática nos ayudará a crecer como ciudadanos que participan de su comunidad. A juicio de Honneth (1998), el concepto de democracia creativa, propuesto por Dewey ya en su madurez, representa una alternativa plausible ante el desfallecimiento de la democracia y su necesaria reactivación. De hecho, se deja notar su impronta en modelos teóricos actualmente celebrados, como el de la cooperación reflexiva y otros. La acción reflexiva, según Guichot (2003) comentando la obra de Dewey, capaci- ta para la planificación consciente, lo que supone una liberación de la rutina, facilita el desarrollo de la técnica a través del pensamiento y sus efectos en la acción, y dota a las cosas de significado, convirtiéndolas en objetos. Dewey (1910/1989), no obstante, apostilla que la reflexión, como la información y la técnica, no son en sí mismas suficientes si no se da una disposición o una actitud personal del sujeto, algo que conocen perfectamente los educadores y saben todos aquellos que han estudiado y constatado, a veces en carne propia como Walter Benjamin, los límites de la "buena educación".

Este protagonismo de las convicciones individuales proporciona una potente dimensión educativa a la democracia. Nada hay más opuesto a este planteamiento que el juicio apriorístico que esconde la expresión "hacer pedagogía" como sinónimo de comunicar con claridad una idea o una medida política, tan recurrente en los análisis coloquiales sobre el distanciamiento entre gobernantes y ciudadanos. Este didactismo, que remeda en el ámbito político y social la relación jerárquica y unívoca maestro-alumno, induce a pensar en la democracia como un aparato legal e institucional en manos de una oligarquía de expertos y no, como propone Dewey, una experiencia de vida compartida, que se nutre de inteligencia social y principios éticos encarnados en cada individuo, gracias a un proceso formativo hecho de vivencia democrática cotidiana. En la obra de John Dewey, como afirma Quay (2016), "democracia y educación van juntas en un sentido más que fundamental como expresiones de vida“ (p. 13). Por eso, la democracia creativa no es tanto una teoría normativa, propia de la ciencia política, como un ideal social ( $y$, por ello, también educativo), tan vivo y deseable en su tiempo como en el nuestro.

\section{AGRADECIMIENTOS}

Agradecemos a Miguel A. Pereyra sus comentarios, que han enriquecido notablemente este artículo.

\section{NOTAS}

1. A este respecto puede consultarse el capítulo "Cuestiones educativas" de la obra Los libros que nunca he escrito de George Steiner (2008, pp. 145-183). Es recomendable asimismo la lectura de su obra Elogio de la transmisión. Maestros y alumnos (2005), en diálogo compartido con Cécile Ladjali, una profesora de literatura en un instituto francés de educación secundaria. En ella ambos subrayan la importancia de una pedagogía exigente y esforzada, la satisfacción de enseñar y de aprender, y la defensa de la cultura humanista, a la que todos pueden y deben acceder.

2. Esta contribución procede de un seminario sobre la figura de John Dewey, celebrado en la Universidad de Valencia, cuyas conclusiones fueron recogidas en una publicación de la Fundación de Investigaciones Educativas y Sindicales, Cuadernos FIES, 1 (abril, 2004), que puede consultarse en: http://pruebas. ccoo.es/comunes/recursos/99914/
pub52277_Democracia_y_Educacion,_ John_Dewey_y_los_viejos_problemas_ educativos.pdf

3. En particular de Max Horkheimer, en su Crítica de la razón instrumental (1969).

4. Véase, en relación con la recepción y difusión internacional de la obra de John Dewey, el volumen monográfico 3 (2) de la revista Espacio, Tiempo y Educación (2016). Con este monográfico la revista conmemoraba el centenario de Democracia y educación. 
5. Domingo Barnés, Ministro de Instrucción Pública durante la II República (1933) y Profesor de Pedagogía en la Escuela Superior de Magisterio, fue secretario y director del Museo Pedagógico Nacional y colaboró con la Institución Libre de Enseñanza. Junto a otro institucionista, Lorenzo Luzuriaga, tradujo y divulgó gran parte de obra de Dewey en España. Barnés hace una primera traducción de $L a$ escuela y la sociedad, que se publicó en

\section{BIBLIOGRAFÍA}

Álvarez Junco, J. (2016). Dioses útiles. Naciones y nacionalismos. Barcelona: Galaxia Gutenberg.

Apple, M. W. y Beane, J. A. (comps.) (1997). Escuelas democráticas. Madrid: Morata.

Atkinson, J. C. (2017). Countering the Neos: Dewey and a Democratic Ethos in Teacher Education. Democracy \& Education, 25 (2), pp. 1-11. Disponible en: https://democracyeducationjournal. org/home/vol25/iss2/2/

Barnés, D. (1929). Prólogo. En: Dewey, J. (1899/1929). La escuela y la sociedad (3. a ed.). Madrid: Francisco Beltrán, pp. 5-15.

Bauman, Z. (2007). Los retos de la educación en la modernidad líquida. Barcelona: Gedisa.

Bauman, Z. (2017). Retrotopía. Barcelona: Paidós.

Bauman, Z. y Lyon, D. (2013). Vigilancia líquida. Barcelona: Austral.

Bellatalla, L. (2016). Democracy and Education: perché? Espacio, Tiempo y Educación, 3 (2), pp. 17-30. https://doi. org/10.14516/ete.2016.003.002.001

Beltrán Llavador, F. (2004). Un encuentro con John Dewey. Cuadernos FIES, 1, pp. 2-5.

Bernstein, R. (2010). Filosofía y democracia: John Dewey. Barcelona: Herder.

Cadrecha Caparrós, M. A. (1990). John Dewey: Propuesta de un modelo educativo. I. Fundamentos. Aula Abierta, 55, pp. 61-87.

Camps, V. (1990). Ética y democracia. Una ética provisional para una democracia imperfecta. Revista del Centro de Estudios Constitucionales, 6, pp. 25-35.

Castillo, R. del (2003). Introducción. El amigo americano. En Dewey, J. (1930/2003). Viejo y nuevo individualismo. Barcelona: Paidós, pp. 9-52.
1915 con el título La escuela y el progreso social en el Boletín de la Institución Libre de Enseñanza (XXXIX, 662, pp. 129-134, y 663, pp. 161-165). Para más información, véase Pereyra (1979). Sobre la influencia de la obra de Dewey en la pedagogía española del primer tercio del siglo $X X$, véase Jover (2010) y Jover y del Pozo (2013).

6. Baste recordar las luminosas y conocidas palabras de Rousseau en su Contra-

Chanial, Ph. (2006). Une foi commune : démocratie, don et éducation chez John Dewey. Revue du MAUSS, 28, pp. 205-250. https://doi.org/10.3917/ rdm.028.0205

Dewey, J. (1897/1997). Mi credo pedagógico. León: Universidad de León. Disponible en http://presencias.net/educar/ ht1050b.html

Dewey, J. (1899/1929). La escuela y la sociedad (3. ed.). Madrid: Francisco Beltrán.

Dewey, J (1910/1989). Cómo pensamos. Barcelona: Paidós.

Dewey, J. (1916/1995). Democracia y educación. Madrid: Morata.

Dewey, J. (1927/2004). La opinión pública y sus problemas. Madrid: Morata.

Dewey, J. (1930/2003). Viejo y nuevo individualismo. Barcelona: Paidós.

Dewey, J. (1935/1996). Liberalismo y acción social. Valencia: Fundación Alfonso el Magnánimo.

Dewey, J. (1938/2004). Experiencia y educación. Madrid: Biblioteca Nueva.

Dewey, J. (1938/2006). La démocratie créatrice. La tâche que nous attend. Revue du MAUSS, 28, pp. 251-256. https://doi. org/10.3917/rdm.028.0251

Dewey, J. (1939/1965). Libertad y cultura México: Uteha.

Dewey, J. (2009). Democracia y escuela. Madrid: Editorial Popular.

Fukuyama, F. (2019). Identidad. La demanda de dignidad y las políticas de resentimiento. Barcelona: Ediciones Deusto / Editorial Planeta.

Geneyro, J. C. (1991). La democracia inquieta: E. Durkheim y J. Dewey. Barcelona: Anthropos.

Giroux, H. (2006). La escuela y la lucha por la ciudadanía. México: Siglo XXI. to social: "El hombre llega a ser hombre sólo cuando se ha hecho ciudadano" (véase Rubio Carracedo, 2009, p. 278).

7. Gaceta de Madrid, no 273,30 de septiembre de 1931, p. 2091.

8. Publicada muchos años después, en 1997 y posteriormente en 2006, con el título La démocratie créatrice. La tâche que nous attend.

Good, J. y Garrison, J. (2010). Dewey, Hegel, and Causation. The Journal of Speculative Philosophy, 24 (2), pp. 101-120. https://doi.org/10.5325/ jspecphil.24.2.0101

González Monteagudo, J. (2001). John Dewey y la pedagogía progresista. En: Trilla, J. (coord.). El legado pedagógico del siglo XX para la escuela del siglo XXI. Barcelona: Graó, pp. 15-40.

Gordon, M. y English, A. R. (2016). John Dewey's Democracy and Education in an Era of Globalization. Educational Philosophy and Theory, 48 (10), pp. 977980. https://doi.org/10.1080/0013185 7.2016.1204742

Guichot, V. (2003). Democracia, ciudadanía y educación. Madrid: Biblioteca Nueva.

Guttman, A. (2001). La educación democrática: Una teoría política de la educación. Barcelona: Paidós.

Han, B.-Ch. (2012). La sociedad del cansancio. Barcelona: Herder.

Han, B.-Ch. (2013). La sociedad de la transparencia. Barcelona: Herder.

Han, B.-Ch. (2014). En el enjambre. BarceIona: Herder.

Han, B.-Ch. (2015a). Psicopolítica. Barcelona: Herder.

Han, B.-Ch. (2015b). El aroma del tiempo. Un ensayo filosófico del arte de demorarse. Barcelona: Herder.

Han, B.-Ch. (2017). La expulsión de lo distinto. Barcelona: Herder.

Hegel, G. W. F. (1821/1968). Filosofía del Derecho (5.a ed). Buenos Aires: Editorial Claridad.

Honneth, A. (1998). Democracy as Reflexive Cooperation. John Dewey and the Theory of Democracy Today. Political Theory, 26 (6), pp. 763-783. https://doi. org/10.1177/0090591798026006001 
Hook, S. (2000). John Dewey. Semblanza intelectual. Barcelona: Paidós.

Horkheimer, M. (1969). Crítica de la razón instrumental. Buenos Aires: Sur.

Igelmo, J., Fuentes, J. L. y Jover, G. (2017). Tres lecturas de John Dewey desde las teorías de la desescolarización. Crónica. Revista científico profesional de la Pedagogía y Psicopedagogía, 2, pp. 25-37.

Jover, G. (2010). Readings of the Pedagogy of John Dewey in Spain in the Early Twentieth Century. Reconciling Pragmatism and Transcendence. En: Bruno-Jofré, R., Johnston, J. S., Jover, G. y Thröhler, D. (eds.). Democracy and the Intersection of Religion and Traditions. The Reading of John Dewey's Understanding of Democracy and Education. Montreal: McGill-Queen's University Press, pp. 79-130.

Jover, G. y García Fernández, A. (2015). Relectura de la educación por competencias desde el pragmatismo de John Dewey. Education in the Knowledge Society, 16 (1), pp. 32-43. https://doi. org/10.14201/eks20151613243

Jover, G. y Pozo, M. M. del (2013). Presencia e influencia de John Dewey en la pedagogía española del primer tercio del siglo XX. Rassegna di Pedagogia, 71 (1-2), pp. 55-74.

Jover, G., Ruiz-Valdepeñas, M. A. y Thoilliez, B. (2010). La obra de John Dewey en su tránsito por España durante el primer tercio del siglo XX. Dos ejemplos de recepción editorial. Edetania, 37, pp. 33-56.

Klenner, A. (2002). En torno a la filosofía del derecho de Hegel: cuestiones y objeciones. Polis. Revista Latinoamericana, 3. Disponible en http://polis.revues. org/7735

Lledó, E. (2014). Los libros y la libertad (4. ed.). Barcelona: RBA.

Luzuriaga, L. (1968). Ideas pedagógicas del siglo XX. Buenos Aires: Losada.
Nubiola, J. y Sierra, V. (2001). La recepción de Dewey en España y Latinoamérica. Utopía y Praxis Latinoamericana. Revista Internacional de Filosofía Iberoamericana y Teoría Social, 6 (13), pp. 107-119.

Nussbaum, M. C. (2010). Sin fines de lucro. Por qué la democracia necesita de las humanidades. Madrid: Katz editores.

Pereyra, M. A (1979). El principio de actividad en John Dewey y en la Institución Libre de Enseñanza: Un estudio comparado. Revista Española de Pedagogía, 37 (144), pp. 79-94.

Popkewitz, Th. (2003). Dewey y Vygotsky: ideas en espacios históricos. En: Popkewitz, Th., Franklin, B. y Pereyra, M. A. (comps.). Historia cultural y educación. Ensayos críticos sobre conocimiento y escolarización. Barcelona: Pomares, pp. 359-400.

Popkewitz, Th. (2008). Inventing the Modern Self and John Dewey. New York: Palgrave.

Putnam, H. y Putnam, R. (1993). Education for Democracy. Educational Theory, 43 (4), pp. 361-376. https://doi. org/10.1111/j.1741-5446.1993.00361.x

Quay, J. (2016). Not 'democratic education' but 'democracy and education': Reconsidering Dewey's oft misunderstood introduction to the philosophy of education. Educational Philosophy and Theory, 48 (10), pp. 1013-1028. https://doi. org/10.1080/00131857.2016.1174098

Rader, M. (1975). Ética y democracia. Navarra: Verbo Divino.

Rawls, J. (1996). El liberalismo político. Barcelona: Crítica.

Ríos, F. de los (1926/1976). El sentido humanista del socialismo. Madrid: Castalia.

Rogacheva, Y. (2016). The Reception of John Dewey's Democratic Concept of School in Different Countries of the World. Espacio, Tiempo y Educación, 3
(2), 65-87. https://doi.org/10.14516/ ete.2016.003.002.003

Rorty, R. (2000). Introducción. En: Hook, S. (ed.). John Dewey. Semblanza intelectual. Barcelona: Paidós, pp. 11-17.

Rubio Carracedo, J. (2009). Educar ciudadanos: el planteamiento republicano-liberal de Rousseau. En: Rubio Carracedo, J., Rosales, J. M. y Toscano, M. (coords.). Democracia, ciudadanía y educación. Madrid: Akal, pp. 277-296.

Sarofian-Butin, D. W. (2017). Democracy Dies in Dualisms. A Response to "Dewey and Democracy". Democracy \& Education, 25 (2). Disponible en: https:// scholarworks.merrimack.edu/soe facpub/97/

Sartori, G. (2007). ¿Qué es la democracia? Madrid: Taurus.

Sousa Santos, B. de (2006). Globalización y democracia. Archipiélago, 73-74, pp. 111-125.

Steiner, G. (2008). Los libros que nunca he escrito. Madrid: Siruela.

Steiner, G. (2012). La idea de Europa (4.a ed.). Madrid: Siruela.

Steiner, G. y Ladjali, C. (2005). Elogio de la transmisión. Maestros y alumnos. Madrid: Siruela.

Tolentino Mendonça, J. (2017). Pequeña teología de la lentitud. Barcelona: Fragmenta Editorial.

Vaamonde Gamo, M. y Nubiola, J. (2016). El legado feminista de John Dewey. Espacio, Tiempo y Educación, 3 (2), pp. 281-300. https://doi.org/10.14516/ ete.2016.003.002.012

Waddington, D. I. (2008). John Dewey: Closet Conservative? Paideius, 17 (2), pp. 51-63. 